\title{
Cristallisation fractionnée de la matière grasse laitière anhydre
}

\author{
par \\ G. LECHAT*, P. VARCHON*, S. KUZDZAL-SAVOIE**, \\ D. LANGLOIS** et W. KUZDZAL ${ }^{* *}$
}

\section{I. - INTRODUCTION}

La matière grasse laitière anhydre (MGLA) peut être fabriquée à partir du beurre après fusion, centrifugation et séchage sous vide de celui-ci. Elle est plus généralement obtenue à partir de crème soumise à une action mécanique et thermique de déstabilisation, à des centrifugations successives et à un séchage sous vide.

Les propriétés physiques de la MGLA dépendent de sa composition chimique. Constituée presque exclusivement de glycérides (9697 p. 100 de triglycérides, 2-3 p. 100 de diglycérides, 0,1 p. 100 de monoglycérides), la MGLA ne contient que des traces de phospholipides, mais renferme 0,5 p. 100 environ de composés liposolubles ( $\beta$-carotène et autres hydrocarbures naturels, cholestérol, vitamines, etc.).

Les acides gras représentent donc $85-90$ p. 100 de la MGLA. La nature même de ces acides gras, leurs proportions relatives et leur répartition dans les molécules de triglycérides déterminent finalement les propriétés physiques de la MGLA, phase continue sans structure globulaire. Or les conditions qui existent au niveau même de la production du lait. les conditions d'alimentation des vaches laitières en particulier, déterminent la composition en acides gras de la MGLA.

Ainsi le transformateur laitier n'est pas maître de la matière première.

Le manque de «tartinabilité » en hiver de certains beurres, l'aspect huileux de certains autres en été peuvent être des causes d'une réduction de la consommation. En outre, il est pratiquement impossible de trouver actuellement sur le marché un beurre qui s'étale correctement dès la sortie du réfrigérateur ménager.

* Service Recherche et Développement, Société Préval.

** Station Centrale de Recherches Laitières et de Technologie des Produits Animaux, I.N.R.A. - 78350 Jouy-en-Josas. 
Par ailleurs, la MGLA, appréciée par les avantages qu'elle apporte sur le plan organoleptique, ne possède pas toujours les qualités pâtissières souhaitées. Enfin, les nutritionnistes reprochent parfois à la MGLA d'être pauvre en acides gras " essentiels » et ceci en toute saison.

Le fractionnement de la MGLA constitue un moyen permettant de modifier la composition en acides gras de telle façon que les propriétés physiques de la MGLA se situent hors des limites normalement observées.

La cristallisation fractionnée de la MGLA est un procédé qui permet d'obtenir des fractions de MGLA très différenciées. Différents systèmes ont été utilisés. Certains sont déjà exploités dans l'industrie, d'autres restent encore du domaine du laboratoire.

La cristallisation de la MGLA a fait l'objet de diverses études poursuivies par Mulder aux Pays-Bas, de Man au Canada, Antila en Finlande, Sherbon aux Etats-Unis, Dolby en Nouvelle-Zélande, Vergelesov en U.R.S.S. (cf. Webb, 1965 ; Antila, 1966 ; Vergelesov, 1970 ; Norris et al., 1971 ; Sherbon, 1974).

Ces dernières années, un effort de développement a été entrepris et une exploitation sur le plan pratique du procédé de cristallisation fractionnée de la MGLA a conduit à l'obtention de fractions de MGLA très diversifiées et possédant des caractéristiques physico-chimiques très remarquables ouvrant des perspectives nouvelles d'utilisation.

La cristallisation simple ou normale, réalisée au sein même de la masse de MGLA pure se distingue de la cristallisation de la MGLA dissoute dans un solvant. Si dans le second cas, la séparation des phases cristallisées et liquides ne pose pas de problèmes, dans le premier cas, ce stade constitue le point délicat du procédé. Pour combattre la difficulté d'extraire correctement la phase liquide des réseaux de phase solide, le procédé Alfa-Laval préconise l'utilisation d'un composé détergent et d'un électrolyte, qui, ajoutés au mélange cristallisé, " mouillent » les cristaux de phase solide et les rendent plus hydrophiles (cf. Fjaervoll, 1970 ; Jebson, 1970).

La cristallisation simple peut être effectuée de différentes manières. Ainsi la MGLA peut être refroidie à $25^{\circ} \mathrm{C}, 28^{\circ} \mathrm{C}$ ou $30^{\circ} \mathrm{C}$ en un temps défini ( $16 \mathrm{~h}, 32 \mathrm{~h}$ ou $48 \mathrm{~h}$ même) puis, après séparation des phases, la phase liquide peut à nouveau être refroidie à $18^{\circ} \mathrm{C}$ ou $20^{\circ} \mathrm{C}$ et ainsi de suite. Il est également possible de procéder à la cristallisation simple par remontée graduelle de la température. Ainsi Baker (1970) isole une fraction liquide à $13^{\circ} \mathrm{C}$ (par pression de la masse cristallisée) puis élève jusqu'à $30^{\circ} \mathrm{C}$ la température à laquelle est soumise la fraction solide. Il obtient ainsi une fraction liquide à $13^{\circ} \mathrm{C}$, une fraction solide à $30^{\circ} \mathrm{C}$ et une fraction, pondéralement la plus importante, très voisine, quant à la composition en acide gras et aux propriétés physico-chimiques, de la MGLA de départ, quoique moins plastique. 
La technique de la cristallisation fractionnée de la MGLA pourrait sans doute profiter des installations modernes décrites par Coenen en 1974 ou même par Blanc (1969) ou Bouffard (1974).

Les résultats des études antérieures montrent une grande variabilité de composition des fractions. Dans le cas d'un fractionnement en deux phases, l'une solide, l'autre liquide, à une température donnée, un point de ramollissement de 28,2 de la MGLA d'origine conduit à des points de ramollissement de 22,1 pour la phase liquide et de 38,0 pour la phase solide (Fjaervoll, 1970). L'indice d'iode varie plus modérément : de 37,5 à l'origine, il passe à 39,4 et 33,7 dans les fractions.

Baker (1970) obtient une plus grande différenciation du point de fusion puisqu'il regroupe 40 p. 100 de la MGLA de départ en une fraction intermédiaire. La fraction liquide à $13^{\circ} \mathrm{C}$ possède un indice d'iode de 46 , la fraction solide à $30^{\circ} \mathrm{C}$ possède un indice d'iode de 30. Dolby (1970) dispose d'une fraction dont le point de fusion atteint $43,5^{\circ} \mathrm{C}$ après deux cristallisations successives.

La cristallisation de la MGLA dissoute dans un solvant a fait l'objet d'études de laboratoire. Ainsi Antila (1966) a procédé au fractionnement de la MGLA dissoute dans l'acétone.

Pour une proportion de matière grasse et d'acétone de 1 à 3 le temps de cristallisation était de $24 \mathrm{~h}$. La phase solide était séparée par filtration et lavage à l'acétone. Trois cristallisations successives à $-9^{\circ} \mathrm{C}, 0^{\circ} \mathrm{C}$ et $10^{\circ} \mathrm{C}$ ont permis d'obtenir quatre fractions : trois fractions respectivement liquides dans l'acétone à $-9^{\circ} \mathrm{C}$, à $0^{\circ} \mathrm{C}$, à $10^{\circ} \mathrm{C}$ et une fraction solide dans l'acétone à $10^{\circ} \mathrm{C}$.

Les fractions obtenues par cristallisation dans l'acétone et analysées par Antila (1966) sont plus différenciées que les fractions obtenues au cours de la cristallisation simple de la MGLA pure par les auteurs précédemment cités (tab. 1).

La MGLA est constituée de triglycérides mixtes ; le nombre d'atomes de carbone des chaînes triglycéridiques s'étage de 26 à 54 atomes de carbone. Il existe dans la MGLA deux groupes de triglycérides : les triglycérides à longue chaîne constitués surtout par des associations oléique-palmitique-stéarique et les triglycérides à chaîne plus courte renfermant plus généralement acides à courte chaîne et acides insaturés.

La répartition des acides gras sur les trois positions de la molécule de glycérol n'obéit pas à une loi statistique. Il existe une répartition préférentielle des acides gras. Ainsi les acides comptant 4 à 10 atomes de carbone occupent le plus souvent la position 3 de la molécule de glycérol ; en position 1, il est observé une plus forte proportion d'acide stéarique et d'acide oléique que sur les autres positions. L'acide palmitique occupe généralement la position 2 dans les triglycérides à haut poids moléculaire, mais la position 1 
TABLEAU 1

Cristallisation de la MGLA dans l'acétone (selon Antila, 1966)

\begin{tabular}{|c|c|c|c|c|c|c|}
\hline & \multirow{2}{*}{$\begin{array}{l}\text { Indice } \\
\text { d'iode }\end{array}$} & \multirow{2}{*}{$\begin{array}{l}\text { Point de } \\
\text { fusion }\end{array}$} & \multicolumn{4}{|c|}{ Proportions relatives des acides } \\
\hline & & & $14: 0$ & $16: 0$ & 18:0 & $18: 1$ \\
\hline \multicolumn{7}{|l|}{ MGLA d'été } \\
\hline Liquide à $-9^{\circ} \mathrm{C}$ & 50 & 6 & 7,4 & 17,0 & 6,9 & 31,9 \\
\hline Solide à $+10^{\circ} \mathrm{C}$ & 18 & 40 & 12,2 & 33,8 & 18,0 & 11,1 \\
\hline MGLA d'hiver & & & & & & \\
\hline Liquide à $-9^{\circ} \mathrm{C}$ & 43 & 6 & 11,6 & 19,4 & 3,1 & 22,6 \\
\hline Solide à $+10^{\circ} \mathrm{C}$ & 14 & 40 & 13,1 & 44,7 & 10,3 & 5,7 \\
\hline
\end{tabular}

Remarque: Les valeurs relatives à l'indice d'iode et au point de fusion sont déduites d'une représentation graphique.

ou 3 dans les triglycérides à bas poids moléculaire (Kuksis et al., 1972). L'acide myristique occupe préférentiellement la position 2.

$\mathrm{Au}$ total la saturation est globalement plus importante en position 2.

Le taux de triglycérides saturés varie avec la saison (Bejambes et al., 1950). Ce taux atteint en juillet sa valeur la plus élevée en Normandie, mais dans les régions de l'Est, le taux des triglycérides saturés est particulièrement élevé au cours des mois de décembre et janvier.

L'interestérification accroît le point de fusion de la MGLA (De Man, 1961). Or, l'interestérification modifie, et ne modifie que la répartition des acides gras sur la molécule de glycérol. La structure glycéridique détermine donc partiellement les propriétés physicochimiques de la MGLA.

La cristallisation simple de la MGLA ou la cristallisation de la MGLA dissoute dans un solvant ne modifie ni la composition globale des acides gras, ni la structure glycéridique.

Le travail que nous avons entrepris avait pour objectif de préciser la composition en acides gras et en triglycérides des fractions à haut point de fusion et à bas point de fusion obtenues à partir d'une MGLA de composition connue, et ceci dans les conditions de cristallisation simple et de cristallisation dans l'acétone. 


\section{II. - MATERIEL Et MEtHOdeS}

Nous avons procédé à des essais de cristallisation simple, en partant généralement d'une matière grasse laitière relativement insaturée (MGLA d'octobre, de Normandie). Nous avons procédé soit à une seule opération de cristallisation, soit à deux, soit même à trois et quatre cristallisations successives, afin d'obtenir une différenciation plus accentuée de la fraction à plus bas point de fusion.

Nous avons également procédé à la cristallisation dans l'acétone de la MGLA. Nous avons reproduit le protocole expérimental suivi par Antila (1966) : dilution de la MGLA dans trois fois son volume d'acétone, première cristallisation à $-9^{\circ} \mathrm{C}$, deuxième cristallisation à $0^{\circ} \mathrm{C}$, troisième cristallisation à $10^{\circ} \mathrm{C}$. La filtration est effectuée sur verre frité $n^{\circ} 3$ et sous pression réduite. Les cristaux sont lavés à l'acétone. Chaque période de cristallisation dure $24 \mathrm{~h}$.

L'analyse des acides gras par chromatographie en phase gazeuse a été effectuée sur chaque fraction isolée. Les acides comptant au moins 8 atomes de carbone ont été transformés en esters méthyliques par transestérification alcaline et analysés dans des conditions isothermiques à $190^{\circ} \mathrm{C}$ sur colonne de succinate de butanediol. Les acides butyrique et caproïque ont été dosés par la méthode aux savons de baryum (Kuzdzal-Savoie et Kuzdzal, 1968), préconisant une analyse directe par chromatographie en phase gazeuse à $145^{\circ} \mathrm{C}$ sur colonne de succinate de diéthylène glycol additionné d'acide phosphorique ou sur colonne de carbowax $20 \mathrm{M}$ réticulé avec de l'acide nitrotéréphtalique (FFAP).

La composition glycéridique a été déterminée par analyse directe des triglycérides par chromatographie en phase gazeuse sur colonne de OV 1 ( 3 p. 100 OV 1 sur chromosorb W ou gaz chromosorb Q). L'indice d'iode a été quelquefois déterminé, ainsi que le taux de cholestérol, ou l'acidité libre.

\section{III. - RESULTATS}

Nous avons rassemblé à titre d'exemple dans le tableau 2 quelques résultats se rapportant à deux essais de cristallisation simple et comportant deux ou quatre cristallisations successives.

La différenciation des fractions est nette. Le rapport oléiquestéarique de l'ordre de 4 dans les fractions liquides s'abaisse à près de 1 dans la fraction solide. Le taux d'acide palmitique est très faible dans la fraction d'indice d'iode 52, mais la MGLA de départ était 
TABLEAU 2

Proportions relatives des acides gras dans la MGLA et dans les fractions (cristallisation simple)

\begin{tabular}{|c|c|c|c|c|c|}
\hline \multirow{3}{*}{ Acides } & \multicolumn{2}{|c|}{ Septembre 1971} & \multicolumn{3}{|c|}{ Février 1972} \\
\hline & \multicolumn{2}{|c|}{ Deux cristallisations successives } & \multicolumn{3}{|c|}{ Quatre cristallisations successives } \\
\hline & MGLA d'origine & $\begin{array}{l}\text { Fraction } \\
\text { la plus liquide }\end{array}$ & MGLA d'origine & $\begin{array}{l}\text { Fraction } \\
\text { la plus liquide }\end{array}$ & $\begin{array}{l}\text { Fraction } \\
\text { la plus solide }\end{array}$ \\
\hline & & & $\mathrm{II}=39,5$ & $\mathrm{II}=52$ & $\mathrm{II}=30,5$ \\
\hline $4 \cdot 0$ & 33 & 40 & 31 & 1 & \\
\hline $6: 0$ & 2,0 & $\begin{array}{l}4,0 \\
26\end{array}$ & $\begin{array}{l}3,1 \\
16\end{array}$ & $\begin{array}{l}4,1 \\
27\end{array}$ & $\begin{array}{l}1,8 \\
08\end{array}$ \\
\hline $8: 0$ & 1,4 & $\begin{array}{l}2,0 \\
1,3\end{array}$ & 1,3 & $\begin{array}{l}2,1 \\
1,6\end{array}$ & $\begin{array}{l}0,0 \\
0,9\end{array}$ \\
\hline $10: 0$ & 2,6 & $\begin{array}{l}1,3 \\
2,8\end{array}$ & 2,3 & 3,0 & 1,9 \\
\hline $10: 1$ & 0,4 & 0,4 & 0,3 & 0,5 & 0,2 \\
\hline $11: 0$ & 0,1 & 0,1 & - & - & - \\
\hline $12: 0$ & 3,0 & 3,3 & 2,8 & 3,2 & 2,5 \\
\hline $12: 1$ & 0,1 & 0,2 & 0,1 & 0,1 & 0,1 \\
\hline i $13: 0$ & 0,1 & 0,2 & 0,2 & 0,1 & 0,1 \\
\hline 13:0 & 0,1 & 0,1 & 0,2 & 0,1 & 0,1 \\
\hline i $14: 0$ & 0,1 & 0,1 & 0,2 & 0,2 & 0,2 \\
\hline 14:0 & 10,8 & 9,7 & 9,5 & 8,5 & 10,0 \\
\hline $14: 1$ & 1,4 & 1,3 & 0,9 & 1,1 & 0,6 \\
\hline i $15: 0$ & 0,3 & 0,4 & 0,5 & 0,6 & 0,4 \\
\hline ai $15: 0$ & 0,6 & 0,7 & 0,8 & 0,8 & 0,7 \\
\hline $15: 0$ & 1,5 & 1,4 & 1,6 & 1,2 & 1,7 \\
\hline $15: 1$ & 0,1 & 0,1 & 0,1 & 0,1 & tr. \\
\hline i $16: 0$ & 0,2 & 0,2 & 0,5 & 0,2 & 0,4 \\
\hline 16:0 & 23,8 & 20,0 & 22,7 & 15,7 & 28,1 \\
\hline $16: 1$ & 2,5 & 2,7 & 1,6 & 2,8 & 1,6 \\
\hline i $17: 0$ & 0,6 & 0,7 & 0,5 & 0,5 & 1,0 \\
\hline ai $17: 0$ & 0,6 & 0,5 & 0,6 & 0,5 & 0,9 \\
\hline $17: 0$ & 1,1 & 0,8 & 1,0 & 0,9 & 1,5 \\
\hline $17: 1$ & 0,2 & 0,3 & 0,5 & 0,6 & 0,5 \\
\hline i $18: 0$ & tr. & tr. & tr. & tr. & tr. \\
\hline 18:0 & 12,3 & 8,7 & 13,6 & 8,1 & 18,8 \\
\hline $18: 1$ & 26,8 & 32,6 & 28,3 & 35,5 & 21,5 \\
\hline $18: 2$ & 2,7 & 3,3 & 2,9 & 3,9 & 2,2 \\
\hline $19: 0$ & tr. & 0,1 & 0,1 & 0,1 & tr. \\
\hline $18: 3$ & 0,6 & 0,6 & 1,1 & 1,6 & 0,7 \\
\hline $18: 2 \mathrm{cj}$ & 0,7 & 0,8 & 1,1 & 1,6 & 0,8 \\
\hline
\end{tabular}




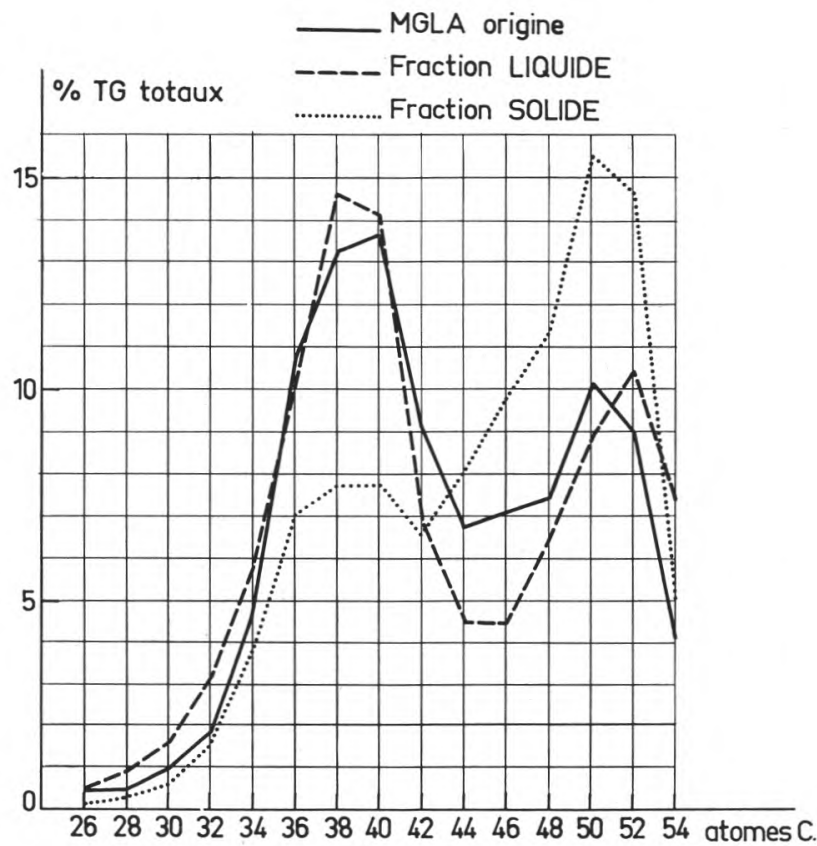

fig. 1

Composition triglycéridique de la MGLA d'origine, de la fraction " la plus liquide " et de la fraction "la plus solide ", obtenues par cristallisation normale (février 1972).

\section{TABLEAU 3}

Résultats du fractionnement par cristallisation dans l'acétone (résultats exprimés en p. 100 de la matière grasse cie départ)

\begin{tabular}{c|c|c|c}
\hline & Essai n $\mathrm{n}^{\circ}$ & Essai n & Essai n $\mathrm{n}^{\circ} 3$ \\
\cline { 2 - 3 } MGLA & 100 & 100 & 100 \\
Liquide à $-9^{\circ} \mathrm{C}$ & 57 & 55 & 43 \\
Solide à $-9^{\circ} \mathrm{C}$ & 43 & 45 & 57 \\
Liquide à $\quad 0^{\circ} \mathrm{C}$ & 16 & 24 & 26 \\
Solide à $\quad 0^{\circ} \mathrm{C}$ & 27 & 21 & 31 \\
Liquide à $+10^{\circ} \mathrm{C}$ & 6,5 & 10 & 13 \\
Solide à $+10^{\circ} \mathrm{C}$ & 20,5 & 11 & 18 \\
\hline
\end{tabular}




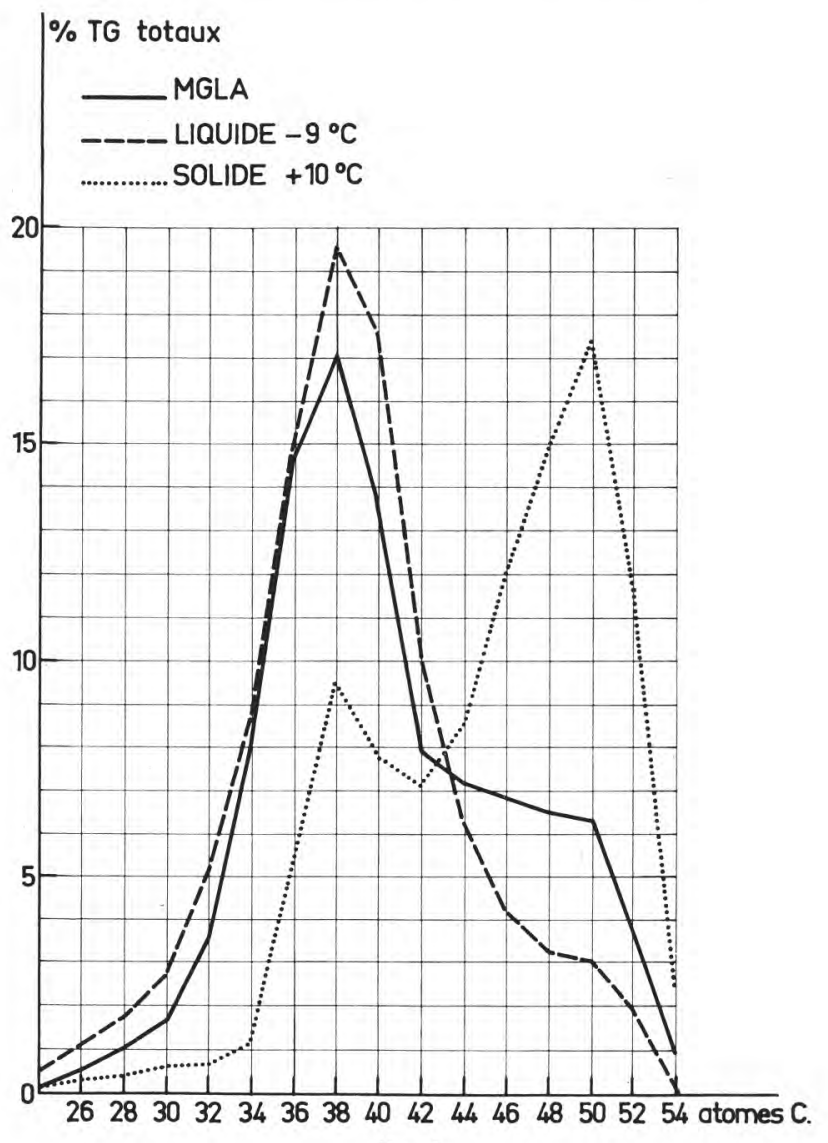

fig. 2

Composition triglycéridique de la MGLA d'origine de la fraction "la plus liquide " (liquide à $-9^{\circ} \mathrm{C}$ ) et de la fraction " la plus solide " (solide à $+10^{\circ} \mathrm{C}$ ) obtenues au cours de la cristallisation fractionnée de la MGLA en solution dans l'acétone (essai 1: septembre 1971).

elle-même relativement insaturée. Les taux des acides à courte chaîne et des acides polyinsaturés sont plus élevés dans les fractions liquides que dans la MGLA d'origine.

Il est bien évident que de tels schémas de répartition des acides gras ne sont jamais rencontrés dans la MGLA normale puisqu'en particulier les acides stéarique et oléique varient toujours parallèlement dans les conditions naturelles.

Les résultats du fractionnement obtenu lors de trois essais de cristallisation dans l'acétone sont rassemblés dans le tableau 3. 


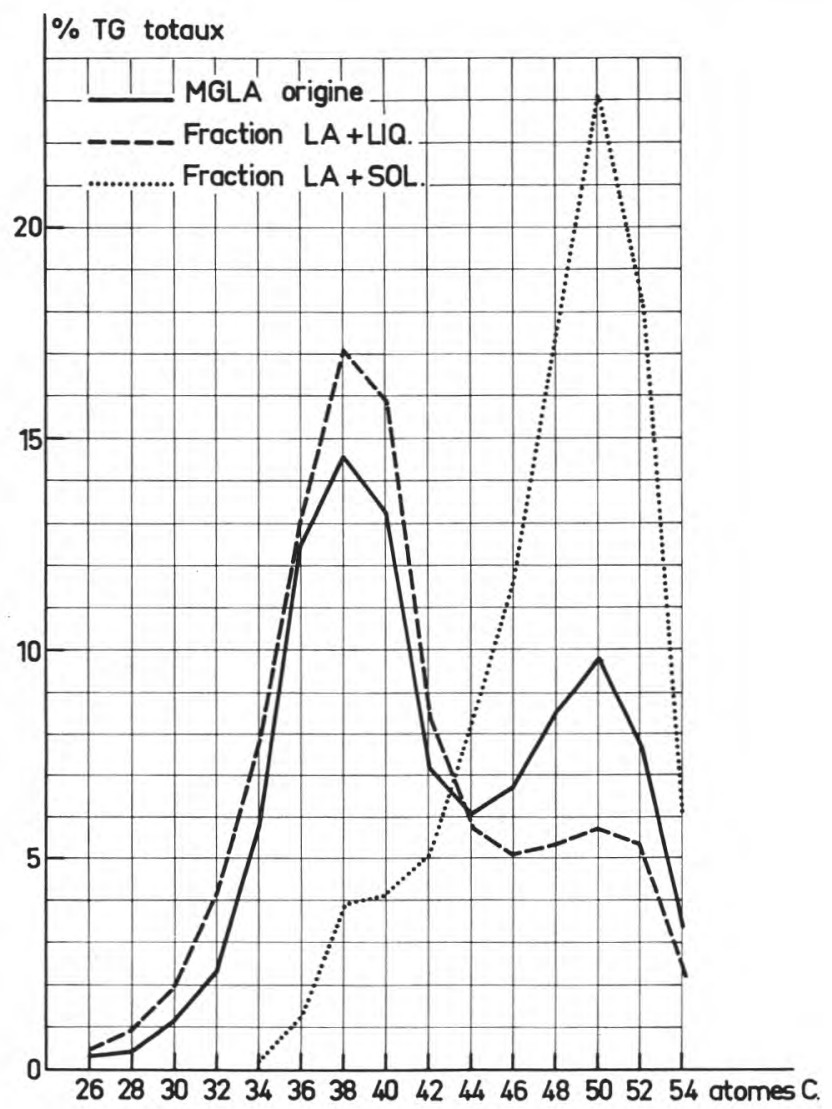

fig. 3

Composition triglycéridique de la MGLA d'origine, de la fraction "la plus liquide " (liquide à $-9^{\circ} \mathrm{C}$ ) et de la fraction " la plus solide " (solide à $+10^{\circ} \mathrm{C}$ ) obtenues au cours de la cristallisations fractionnée de la MGLA en solution dans l'acétone (essai 3: avril 1972).

La fraction encore solide à $10^{\circ} \mathrm{C}$ dans l'acétone représente moins de 20 p. 100 de la MGLA de départ alors que la fraction liquide à $-9^{\circ} \mathrm{C}$ dans l'acétone représente 52 p. 100 en moyenne de la MGLA de départ. Les essais 1 et 2 ont été effectués avec la même MGLA de départ, mais la proportion d'acétone était un peu plus faible dans l'essai $\mathrm{n}^{\circ} 2$ que dans l'essai $\mathrm{n}^{\circ} 1$.

Dans le tableau 4 ( 4 a et 4 b) ont été rassemblés les résultats de l'analyse par chromatographie en phase gazeuse des différentes fractions de MGLA obtenues au cours de deux essais. 
TABLEAU 4 a. - Cristallisation dans l'acétone : essai $\mathrm{n}^{\circ} 1$

Proportions relatives des acides gras (résultats exprimés en g p. 100 de matière grasse)

\begin{tabular}{|c|c|c|c|c|c|c|c|}
\hline \multirow{2}{*}{ Acides gras } & \multirow{2}{*}{$\begin{array}{l}\text { Graisse } \\
\text { de départ }\end{array}$} & \multicolumn{2}{|c|}{ Première cristallisation } & \multicolumn{2}{|c|}{ Deuxième cristallisation } & \multicolumn{2}{|c|}{ Troisième cristallisation } \\
\hline & & Solide (1) & Liquide (2) & Solide (3) & Liquide (4) & Solide (5) & Liquide (6) \\
\hline $4: 0$ & 3,3 & 2,1 & 3,7 & 1,6 & 3,5 & 1,3 & 2,7 \\
\hline $6: 0$ & 2,0 & 1,4 & 2,4 & 1,1 & 2,1 & 0,9 & 1,8 \\
\hline $8: 0$ & 1,4 & 0,9 & 1,3 & 0,6 & 1,2 & 0,5 & 0,9 \\
\hline $9: 0$ & tr. & $\operatorname{tr}$ & tr. & $\operatorname{tr}$ & tr. & tr. & - \\
\hline $10: 0$ & 2,6 & 1,7 & 2,2 & 1,5 & 2,3 & 1,4 & 1,9 \\
\hline $10: 1$ & 0,4 & 0,2 & 0,4 & 0,2 & 0,3 & 0,1 & 0,2 \\
\hline $11: 0$ & 0,1 & 0,1 & - & - & tr. & - & tr. \\
\hline $12: 0$ & 3,0 & 2,4 & 2,6 & 2,0 & 3,1 & 2,0 & 2,0 \\
\hline 12:1 & 0,1 & 0,1 & 0,2 & 0,1 & 0,3 & & \\
\hline i $13: 0$ & 0,1 & 0,1 & 0,2 & 0,1 & 0,2 & & \\
\hline $13: 0$ & 0,1 & 0,1 & 0,1 & tr. & 0,1 & 0,3 & 0,3 \\
\hline i $14: 0$ & 0,1 & 0,1 & 0,2 & tr. & 0,2 & & \\
\hline $14: 0$ & 10,8 & 10,3 & 9,1 & 10,1 & 11,1 & 10,4 & 10,3 \\
\hline $14: 1$ & 1,4 & 1,1 & 1,2 & 0,8 & 1,5 & 0,5 & 0,9 \\
\hline i $15: 0$ & 0,9 & 0,7 & 0,8 & 0,6 & 1,0 & 0,3 & 0,6 \\
\hline 15:0 & 1,5 & 1,6 & 1,3 & 1,9 & 1,7 & 1,5 & 1,7 \\
\hline 15:1 & 0,1 & 0,1 & 0,2 & 0,1 & 0,1 & tr. & 0,1 \\
\hline i $16: 0$ & 0,2 & 0,3 & 0,2 & 0,3 & 0,2 & 0,2 & 0,2 \\
\hline $16: 0$ & 23,8 & 29,5 & 17,6 & 34,4 & 22,8 & 37,4 & 34,8 \\
\hline $16: 1$ & 2,5 & 1,8 & 2,6 & 1,5 & 2,3 & 0,7 & 1,5 \\
\hline i $17: 0$ & 0,6 & 1,1 & 0,8 & 0,7 & 0,8 & 0,6 & 0,5 \\
\hline ai $17: 0$ & 0,6 & 1,0 & 0,7 & 0,7 & 0,7 & 0,4 & 0,3 \\
\hline $17: 0$ & 1,1 & 1,3 & 0,9 & 1,3 & 1,0 & 0,8 & 1,0 \\
\hline $17: 1$ & 0,2 & 0,2 & 0,7 & 0,1 & 0,3 & tr. & tr. \\
\hline i $18: 0$ & tr. & 0,1 & tr. & tr. & tr. & tr. & tr. \\
\hline 18:0 & 12,3 & 18,1 & 7,1 & 23,3 & 10,3 & 27,2 & 20,1 \\
\hline $18: 1$ & 26,8 & 19,2 & 36,3 & 15,1 & 27,6 & 12,7 & 16,2 \\
\hline $18: 2$ & 2,7 & 3,0 & 4,4 & 1,3 & 3,4 & 0,8 & 1,5 \\
\hline $19: 0$ & - & - & 0,1 & 0,2 & 0,1 & - & tr. \\
\hline $18: 3$ & 0,6 & 0,5 & 1,1 & 0,1 & 0,8 & tr. & 0,1 \\
\hline $18: 2 \mathrm{cj}$ & 0,7 & 0,8 & 1,5 & 0,2 & 1,0 & tr. & 0,4 \\
\hline $20: 0$ & - & 0,1 & 0,1 & 0,1 & - & tr. & tr. \\
\hline $20: 1$ & - & tr. & - & tr. & - & - & - \\
\hline
\end{tabular}


TABLEAU 4 b. - Cristallisation dans l'acétone : essai $\mathrm{n}^{\circ} 3$

Proportions relatives des acides gras (résultats exprimés en g p. 100 de matière grasse)

\begin{tabular}{|c|c|c|c|c|c|c|}
\hline \multirow{2}{*}{ Acides gras } & \multirow{2}{*}{$\begin{array}{c}\text { Graisse } \\
\text { de départ }\end{array}$} & Première cristallisation & \multicolumn{2}{|c|}{ Deuxième cristallisation } & \multicolumn{2}{|c|}{ Troisième cristallisation } \\
\hline & & Liquide & Solide & Liquide & Solide & Liquide \\
\hline $4 \cdot 0$ & & & 21 & & & \\
\hline $\begin{array}{l}4.0 \\
6: 0\end{array}$ & 2,0 & $\begin{array}{l}4,1 \\
20\end{array}$ & $\begin{array}{l}2,1 \\
1,4\end{array}$ & 2,0 & 0,5 & 21 \\
\hline $8: 0$ & 1,0 & 1,3 & 0,5 & $\begin{array}{l}2,0 \\
1,9\end{array}$ & 0,2 & $\begin{array}{l}2,1 \\
1,1\end{array}$ \\
\hline 10:0 & 2,6 & 2,4 & 1,6 & 3,3 & 0,9 & 2,0 \\
\hline $10: 1$ & 0,3 & 0,4 & 0,2 & 0,6 & 0,1 & 0,3 \\
\hline $\begin{array}{l}11: 0 \\
12: 0\end{array}$ & 2,9 & 3,0 & 2,3 & 3,8 & 1,8 & 2,3 \\
\hline $12: 1$ & tr. & 0,1 & & 0,1 & $\begin{array}{l}1,0 \\
\text { tr. }\end{array}$ & 2,0 \\
\hline i $13: 0$ & 0,1 & 0,2 & 04 & 0,2 & tr. & 0 \\
\hline i3:0 & tr. & 0,1 & 0,4 & 0,2 & tr. & 0,4 \\
\hline i $14: 0$ & 0,1 & 0,2 & & 0,3 & tr. & \\
\hline $14: 0$ & 9,7 & 8,4 & 10,9 & 11,2 & 9,8 & 9,6 \\
\hline $14: 1$ & 0,9 & 1,3 & 0,7 & 1,2 & 0,8 & 0,8 \\
\hline i $15: 0$ & 0,3 & 0,6 & 0,5 & 0,7 & & \\
\hline ai 15:0 & 0,5 & 1,0 & 0,4 & 1,2 & & 0,6 \\
\hline $15: 0$ & 1,1 & 1,6 & 1,9 & 2,0 & 1,6 & 2,0 \\
\hline $15: 1$ & tr. & - & - & 0,2 & - & - \\
\hline i $16: 0$ & 0,1 & 0,3 & 0,3 & 0,4 & 0,2 & 0,2 \\
\hline 16:0 & 25,3 & 16,5 & 34,0 & 20,8 & 36,4 & 30,4 \\
\hline $16: 1$ & 1,9 & 2,8 & 0,8 & 2,2 & 0,7 & 1,0 \\
\hline i $17: 0$ & 0,6 & 0,9 & 0,8 & 1,1 & 0,9 & 1,0 \\
\hline ai $17: 0$ & 0,6 & 0,9 & 0,7 & 1,1 & 0,8 & 0,7 \\
\hline $17: 0$ & 1,4 & 1,4 & 1,4 & 1,6 & 1,2 & 1,9 \\
\hline $17: 1$ & 0,4 & 0,7 & 0,2 & 0,9 & 0,1 & 0,2 \\
\hline i $18: 0$ & tr. & - & - & - & tr. & 0,1 \\
\hline $18: 0$ & 13,7 & 8,7 & 19,6 & 10,9 & 28,6 & 15,2 \\
\hline $18: 1$ & 26,5 & 32,4 & 17,0 & 21,4 & 12,6 & 22,4 \\
\hline $18: 2$ & 3,0 & 5,0 & 1,8 & 3,6 & 1,8 & 2,0 \\
\hline 19:0 & 0,1 & tr. & - & - & tr. & - \\
\hline $18: 3$ & 0,7 & 1,6 & 0,2 & 1,7 & 0,1 & 0,2 \\
\hline $18: 2 \mathrm{cj}$ & 0,8 & 1,5 & 0,3 & 1,5 & 0,2 & 0,3 \\
\hline
\end{tabular}


On constate une différenciation très prononcée des fractions. Le taux d'acide stéarique s'abaisse à 7-8 p. 100 dans la fraction la plus liquide et s'élève à $27-28 \mathrm{p} .100$ dans la fraction la plus solide. Les trois essais de cristallisation dans l'acétone sont très similaires quant aux résultats relatifs à la composition en acides gras. Le taux d'acide oléique s'élève à 33-36 p. 100 dans la fraction la plus liquide et s'abaisse à 12,6 p. 100 dans la fraction la plus solide. La différenciation concernant les acides volatils est également nette.

Cependant si la différenciation des fractions les plus solides obtenues soit par cristallisation simple, soit par cristallisation dans l'acétone est assez prononcée, la composition en acides gras de la fraction la plus liquide obtenue par cristallisation simple ou par cristallisation dans l'acétone reste assez similaire.

L'analyse de la composition triglycéridique fait apparaître de nouvelles différences. Dans la cristallisation simple, la composition triglycéridique de la fraction la plus liquide se différencie peu de la MGLA de départ. Il arrive par contre que la fraction la plus solide soit nettement différenciée (fig. 1), mais ce n'est pas toujours le cas.

Par contre, la différenciation des fractions, quant à leur composition triglycéridique est très nette lors de la cristallisation dans l'acétone (cf. fig. 2 et 3 ). La fraction à haut point de fusion ne contient qu'une proportion réduite de triglycérides comptant moins de 34 atomes de carbone.

\section{IV. - DISCUSSION}

Le procédé de cristallisation dans l'acétone, bien que signalé en technologie du fractionnement (Coenen, 1974) comme possible, n'est certainement pas à retenir pour le fractionnement de la MGLA. Les servitudes technologiques que ce procédé impose, l'introduction de la " chimie " dans le fractionnement et les risques de résidu qu'il entraîne ne permettent pas de défendre ce procédé. Par ailleurs, il deviendrait diffizile d'admettre que la fraction à haut point de fusion puisse encore être assimilée à une MGLA. Privée de ses acides volatils, de la plus grande partie de l'acide oléique, contenant plus d'acide stéarique que le suif de bœuf, cette fraction est-elle encore une MGLA ? Elle se rapproche effectivement d'un beurre de cacao, et cette similitude l'a fait utiliser avec succès en chocolaterie. Ceci montre l'avantage de fixer des limites de composition pour définir un produit tel que la "fraction à haut point de fusion ".

La cristallisation simple de la MGLA sans addition d'aucun produit chimique, par contre, constitue un procédé technologique à l'abri de toute critique. Même si la différenciation des fractions est 
limitée, elle est cependant suffisante pour que les propriétés physicochimiques de la nouvelle MGLA soient tout à fait originales, ouvrant la possibilité d'utilisation dans des domaines jusqu'ici difficilement accessibles à la MGLA normale ou naturelle. La fraction à haut point de fusion est maintenant déjà appréciée dans la fabrication des pâtes feuilletées.

La fraction liquide peut permettre la fabrication de beurres possédant des propriétés physiques remarquables (bonne tartinabilité à 7,5 $\mathrm{C}$ par exemple, si l'on se réfère aux essais de Dolby, 1970), il est aussi intéressant de remarquer que l'accroissement du taux des acides essentiels confère à cette fraction liquide des propriétés nutritionnelles en accord avec les exigences actuelles de certains nutritionnistes, par exemple la proportion souhaitée de 1 p. 100 des calories totales de la ration en acide linoléique pourrait être satisfaite. Ainsi la fraction liquide de la MGLA pourrait être avantageusement utilisée dans la fabrication de poudres de lait destinées à l'alimentation infantile. D'autant plus que les vitamines liposolubles se concentrent dans la fraction liquide (Norris et al., 1971), comme le fait d'ailleurs le cholestérol d'une façon modérée $(0,4 \mathrm{~g}$ de cholestérol dans une fraction liquide de MGLA). Cependant la plus grande richesse en acides polyinsaturés exige que des précautions accrues soient prises vis-à-vis des risques d'oxydation ou de lipolyse.

Des problèmes de législation peuvent se poser à l'occasion de l'introduction de ces nouveaux produits sur le marché. Si le problème de la fraction liquide n'existe pas pour l'instant (il n'est sans doute pas utile de contrôler sa présence ou de vouloir la distinguer de la MGLA) il s'avère nécessaire de distinguer la fraction à haut point de fusion d'une MGLA normale ou d'autres formes de MGLA (par exemple : MGLA hydrogénée). Il est probable que le taux réduit de triglycérides courts puisse constituer un critère utilisable au cours du contrôle : l'isolement des triglycérides saturés et l'analyse de leur composition en acides gras et en triglycérides fourniraient d'autres éléments d'information sur la nature des MGLA soumises au contrôle. Enfin les modifications atteignant certains composés de l'insaponifiable mériteraient une étude attentive.

\section{Rés u m é}

La cristallisation de la matière grasse laitière anhydre (MGLA) est un procédé qui permet d'obtenir des fractions de MGLA très différenciées quant aux propriétés physiques et quant à la composition en acides gras.

Dans le cas d'une cristallisation simple, sans addition d'aucun produit chimique, la différenciation des fractions est fonction de 
l'efficacité de la séparation. La cristallisation de la MGLA dissoute dans l'acétone est plus facile et plus efficace, mais un tel procédé n'est pas applicable en pratique (risque de résidus).

Le rapport 18:1 / 18:0 généralement supérieur à 2 pour une MGLA normale peut s'abaisser à 1,2 ou même 0,5 dans les fractions les plus solides obtenues respectivement par cristallisation directe de la MGLA ou par cristallisation de la MGLA dissoute dans l'acétone. Ce même rapport 18:1 / 18:0 peut atteindre 3 à 4, selon l'efficacité du fractionnement, dans la fraction liquide obtenue par cristallisation simple et 4 à 5 dans la fraction liquide obtenue par cristallisation dans l'acétone.

Il est donc nécessaire de fixer les limites de composition en acides gras des fractions obtenues.

La technique de fractionnement par cristallisation simple ou directe constitue un moyen de diversifier les utilisations de la MGLA en adaptant celle-ci aux exigences du marché.

\section{S u $\mathrm{m} \mathbf{m}$ a $\mathbf{r} \mathbf{y}$}

The crystallization of anhydrous milk fat is a process which makes it possible to obtain fractions of milk fat which are clearly differentiated in respect to their physical properties and their fatty acid composition.

In the case of a direct crystallization, without the use of any solvents or additives, the differentiation of the fractions is in function to the effectiveness of the separation between solid and liquid phases. In the case of acetone crystallization of milk fat, the separation of the solid and liquid phases is without problem, but, such a process is not applicable in practice (risk of residues).

The ratio 18:1 / 18:0 generally higher than 2 for a normal milk fat may drop to 1.2 or even 0.5 in the first and most solid fraction obtained respectively by direct crystallization or by acetone crystallization of the milk fat. This same ratio 18:1 / 18:0 may reach 3 or 4, according to the effectiveness of the separation, in the liquid fraction obtained by direct crystallization and 4 to 5 in the liquid fraction obtained by acetone crystallization.

Thus it is necessary to fix the limits of the fatty acid and triglyceride compositions of the fractions obtained, specially of the solid fraction.

The technique of fractioning milk fat by direct crystallization offers a means of diversifying the uses of milk fat by adapting it to commercial requirements. 


\section{Références bibliographiques}

ANTILA (V.) (1966). - Fatty acid composition, solidification and melting of finnish butter fat. Meijeritreteellinen Aikakanskirja, 27, 1-72.

BAKER (B. C.) (1970). - Fractionnement des matières grasses et propriétés des fractions sélectionnées. 18 Congr. Intern. Lait. Sydney, A-4-2, p. 247.

Bejambes (M.), Veisseyre (R.), Legrand (M.) (1950). - Un aspect de la question beurrière française. Bull. SPEL., 5, 31-33.

BLANC (I.) (1969). - Techniques modernes de fabrication des margarines. Rev. Franç. Corps Gras, 16, 457-472.

Bouffard (C.) (1974). - Les margarines tartinables : technologie. Rev. Franç. Corps Gras, 21, 351-357.

Coenen (J. N.E.) (1974). - Fractionnement et interestérification des corps gras dans la perspective du marché mondial des matières premières et des produits finis. I. Fractionnement. Rev. Franç. Corps Gras, 21, 343-349.

DolBy (R. M.) (1970). - Propriétés du beurre reconstitué fait à partir de matière grasse fractionnée. 18 Congr. Intern. Lait, Sydney, A-4-2, p. 248.

FJaervoll (A.) (1970). - Fractionnement de la matière grasse du lait. 18 $\mathrm{e}$ Congr. Intern. Lait, Sydney, A-4-2, p. 245.

JEBSON (R. S.) (1970). - Fractionnement de la matière grasse du lait en composants à points de fusion élevés et bas. $18^{\mathrm{e}}$ Congr. Intern. Lait, Sydney, A-4-2, p. 246.

Kuksis (A.), Marai (L.), Myher (J. J.) (1972). - Triglycerides structure of milk fats. J. Am. Oil Chem. Soc., 50, 193-201.

Kuzdzal-Savoie (S.), Kuzdzal (W.) (1968). - Utilisation des sels de baryum en vue du dosage des acides volatils du beurre. Le Lait, 475-476, 155.

Kuzdzal-SavoIE (S.) (1971). - Etude comparée des lipides du lait. Ann. Nutr. Alim., 25, A 225-A 289.

DE MAN (1961). - Physical properties of milk fat. I. Influence of chemical modification. J. Dairy Res., 28, 81-86.

INorris (R.), Gray (I. K.), Mc Dowell (A. K. R.), Dolby (R. M.) (1971). - The chemical composition and physical properties of fractions of milk fat obtained by a commercial fractionation process. J. Dairy Research, 38, 179-191.

SHERbon (J. W.) (1974). - Crystallization and fractionation of milk fat. J. Am. Oil Chem. Soc., 51, 22.

WebB (B. H.), Johnson (A. H.) (1965). - Fundamentals of dairy chemistry. AVI Publ., Westport, Connecticut (U.S.A.).

Vergelesov (V. M.) et al. (1970). - 18 Congr. Intern. Lait. Sydney, A-4-2, p. 212, $214,216$. 